\title{
Bases communes holomorphes: nouvelle extension du théorème de Whittaker
}

\author{
par Nguyen Thanh Van et Patrice Lassere (Toulouse)
}

Résumé. Soient $D$ un ouvert de $\mathbb{C}$ et $E$ un compact de $D$. Moyennant une hypothèse assez faible sur $D$ et $\overline{\mathbb{C}} \backslash E$ on montre que si $\alpha \in] 0,1\left[\right.$ vérifie $\partial D_{\alpha} \subset D \backslash E, D_{\alpha}$ étant l'ouvert de niveau $\{z \in D: \omega(E, D, z)<\alpha\}$, alors toute base commune de $\mathcal{O}(E)$ et $\mathcal{O}(D)$ est une base de $\mathcal{O}\left(D_{\alpha}\right)$.

0. Introduction. Soient $D$ un ouvert de $\mathbb{C}$ et $E$ un compact dans $D$. On désigne par $\mathcal{O}(D)$ et $\mathcal{O}(E)$ les espaces de fonctions holomorphes sur $D$ et $E$ respectivement, munis de leur topologies usuelles. $\omega(E, D, z)$ (ou plus simplement $\omega)$ sera la fonction sousharmonique extrémale $(0,1)$ associée au couple $(E, D)$ :

$$
\omega(E, D, \cdot)=\operatorname{Reg} \sup \left[\sup \left\{u \in \mathcal{S H}(D): u \leq 1,\left.u\right|_{E} \leq 0\right\}\right]
$$

(elle est harmonique sur $D \backslash E$ ). Les bases communes des espaces $\mathcal{O}(D)$ et $\mathcal{O}(E)$ ont été étudiées par de nombreux auteurs (cf. bibliographie), à commencer par les travaux de J. M. Whittaker exposés dans son livre de la collection Borel.

ThÉORÈme DE WhitTaker. Soient $D_{0}$ et $D_{1}$ deux disques concentriques. Toute base commune de $\mathcal{O}\left(D_{0}\right)$ et $\mathcal{O}\left(D_{1}\right)$ est une base de $\mathcal{O}(D)$, pour tout disque concentrique intermédiaire $D$.

A l'aide de l'important critère de Dynin et Mityagin, il est facile (voir $[\mathrm{N}]$, p. 209) de généraliser cet énoncé au cas où le couple $(E, D)$ est régulier :

Si $\partial D$ est régulier pour le problème de Dirichlet et si $\left.\omega\right|_{E} \equiv 0$, alors toute base commune de $\mathcal{O}(D)$ et $\mathcal{O}(E)$ est une base de $\mathcal{O}\left(D_{\alpha}\right)$, où $D_{\alpha}=$ $\{z \in D: \omega(E, D, z)<\alpha\}$, pour tout $\alpha \in] 0,1[$.

1991 Mathematics Subject Classification: 30B99, 30H50.

Key words and phrases: spaces of holomorphic functions, Schauder bases. 
Nous démontrons ici une nouvelle extension du théorème de Whittaker, avec des hypothèses beaucoup plus générales sur le couple $(E, D)$.

DÉfinition. Un ouvert $\Omega$ de $\overline{\mathbb{C}}$ est dit de type $(\star)$ lorsque pour tout compact $X$ de $\Omega$ et toute suite d'ouverts $\left(\Omega_{k}\right)$ vérifiant

$$
X \subset \Omega_{k} \subset \Omega_{k+1}, \quad \bigcup \Omega_{k}=\Omega,
$$

on a

$$
\lim _{k \rightarrow \infty} \omega\left(X, \Omega_{k}, z\right)=\omega(X, \Omega, z), \quad \forall z \in \Omega .
$$

Remarque 0 . Il est facile de voir que $\Omega$ est de type $(\star)$ s'il vérifie $(\star \star)$ : Pour tout compact $X \subset \Omega$ et tout ensemble polaire $Y$, on a l'égalité $\omega(X, \Omega, \cdot)=\omega(X \backslash Y, \Omega, \cdot) .(\star \star)$ est vérifiée lorsque $\Omega$ est un ouvert borné de $\mathbb{C}$, par conséquent elle l'est encore lorsque chaque composante connexe de $\Omega$ est analytiquement isomorphe à un domaine borné de $\mathbb{C}$.

Convention. Dans ce qui suit, $D$ et $\overline{\mathbb{C}} \backslash E$ seront supposés de type $(\star)$. Cette hypothèse implique que $\overline{\mathbb{C}} \backslash D$ et $E$ sont non polaires.

ThÉORÈme 1. Si $\mathcal{O}(D)$ et $\mathcal{O}(E)$ possèdent une base commune $\left(f_{n}\right)$ et si pour un certain $\alpha \in] 0,1\left[\right.$, la frontière de l'ouvert $D_{\alpha}=\{z \in D$ : $\omega(E, D, z)<\alpha\}$ dans $\overline{\mathbb{C}}$ est incluse dans $D \backslash E$ alors $\left(f_{n}\right)$ est une base de $\mathcal{O}\left(D_{\alpha}\right)$.

A l'aide de ce résultat on prouve

ThÉORÈme 2. On suppose que $D$ est un domaine simplement connexe. Si $\mathcal{O}(D)$ et $\mathcal{O}(E)$ possèdent une base commune $\left(f_{n}\right)$, alors $\omega(E, D, \cdot) \equiv 0$ sur $E\left(\right.$ et $\left(f_{n}\right)$ est une base de $\mathcal{O}\left(D_{\alpha}\right)$ pour tout $\left.\alpha \in\right] 0,1[)$.

Ce théorème est à comparer avec un énoncé de Zakharyuta et Kadampatta (cf. [ZK] et remarque à la fin du paragraphe 2).

\section{Démonstration du théorème 1}

(1) Proposition. Soient $D$ et $G$ des ouverts de type $(\star)$ de $\overline{\mathbb{C}}$, E et $F$ des compacts non polaires dans $D$ et $G$ respectivement. Si $\left\{f_{i}(z, \xi)\right\}_{i \in I}$ est une famille de fonctions holomorphes sur $D \times G$ telle que

$$
\forall L \Subset G, \quad \sup _{i \in I}\left\|f_{i}\right\|_{E \times L}<\infty, \quad \forall K \Subset D, \quad \sup _{i \in I}\left\|f_{i}\right\|_{K \times F}<\infty,
$$

alors pour tout $\alpha \in] 0,1\left[,\left\{f_{i}\right\}_{i \in I}\right.$ est une partie bornée de $\mathcal{O}\left(D_{\alpha} \times G_{1-\alpha}\right)$, ò̀ $D_{\alpha}=\{z \in D: \omega(E, D, z)<\alpha\}$ et $G_{1-\alpha}=\{\xi \in G: \omega(F, G, \xi)<1-\alpha\}$. Autrement dit, $\left\{f_{i}\right\}_{i \in I}$ est une partie bornée de $\mathcal{O}(\Omega)$, avec $\Omega=\{(z, \xi) \in$ $D \times G: \omega(E, D, z)+\omega(F, G, \xi)<1\}$. 
Preuve de la proposition. Soient $\left(D_{j}\right),\left(G_{j}\right)$ des suites d'ouverts bornés réguliers de $\mathbb{C}$ tels que

$$
E \subset D_{j} \Subset D, \quad F \subset G_{j} \Subset G, \quad D_{j} \nearrow D, \quad G_{j} \nearrow G .
$$

Puisque $D$ et $G$ sont de type $(\star)$, la proposition est une conséquence immédiate de l'assertion suivante : Soient pour $j$ fixé

$$
\begin{gathered}
\Omega_{j}=\left\{(z, \xi) \in D_{j} \times G_{j}: \omega\left(E, D_{j}, z\right)+\omega\left(F, G_{j}, \xi\right)<1\right\}, \\
X_{j}=\left(E \times G_{j}\right) \cup\left(D_{j} \times F\right) .
\end{gathered}
$$

Alors pour toute fonction $f$ holomorphe sur un voisinage de $\bar{D}_{j} \times \bar{G}_{j}$ on a

$$
\|f\|_{X_{j}} \geq\|f\|_{\Omega_{j}} .
$$

En effet, si l'on pose, pour tout $k$ suffisamment grand, $E_{k}=\{z: \operatorname{dist}(z, E)$ $\leq 1 / k\}$ et $F_{k}=\{\xi: \operatorname{dist}(\xi, F) \leq 1 / k\}$, alors d'après un résultat de Siciak ([S], Lemme 6.1)

$$
\Omega_{j, k}=\left\{(z, \xi) \in D_{j} \times G_{j}: \omega\left(E_{k}, D_{j}, z\right)+\omega\left(F_{k}, G_{j}, \xi\right)<1\right\}
$$

est l'enveloppe d'holomorphie de $X_{j, k}=\left(E_{k} \times G_{j}\right) \cup\left(D_{j} \times F_{k}\right)$. Par conséquent,

$$
\|f\|_{X_{j, k}} \geq\|f\|_{\Omega_{j, k}}
$$

l'assertion en découle en faisant tendre $k$ vers $\infty$.

Remarque. Notre première preuve de cette proposition est plus sophistiquée. Celle-ci nous a été communiquée par J. Siciak.

(2) Rappelons le théorème de dualité de Köthe-Grothendieck. Pour toute partie $\Omega$ de $\mathbb{C}$ le dual fort de $\mathcal{O}(\Omega)$ est isomorphe topologiquement à $\mathcal{O}_{0}(\overline{\mathbb{C}} \backslash \Omega)$, espace des fonctions holomorphes sur $\overline{\mathbb{C}} \backslash \Omega$ telles que $f(\infty)=0$, de la manière suivante :

A toute forme linéaire continue $\mathcal{L}$ sur $\mathcal{O}(\Omega)$ correspond un unique élément $\Phi_{\mathcal{L}}$ de $\mathcal{O}_{0}(\overline{\mathbb{C}} \backslash \Omega)$ défini par

$$
\Phi_{\mathcal{L}}(\xi)=\mathcal{L}\left(z \rightarrow \frac{1}{z-\xi}\right)
$$

et pour toute fonction $f$ de $\mathcal{O}(\Omega)$ on a

$$
\mathcal{L}(f)=\left\langle\Phi_{\mathcal{L}}, f\right\rangle=\frac{1}{2 i \pi} \int_{\gamma} f(\xi) \Phi_{\mathcal{L}}(\xi) d \xi
$$

où $\gamma$ est un cycle convenablement choisi. Soit maintenant $\left(f_{n}\right)$ une base commune de $\mathcal{O}(D)$ et $\mathcal{O}(E)$. Désignons par $\left(\varphi_{n}\right)$ la base duale de $\left(f_{n}\right)$ dans la dualité $\left(\mathcal{O}(E), \mathcal{O}_{0}(\overline{\mathbb{C}} \backslash E)\right)$; elle l'est aussi dans la dualité $\left(\mathcal{O}(D), \mathcal{O}_{0}(\overline{\mathbb{C}} \backslash D)\right)$.

LEMme. Soient $\mathcal{W}$ un ouvert (ou un compact) de $\mathbb{C}$ et $\left(f_{n}, \varphi_{n}\right)$ un système biorthogonal dans $\mathcal{O}(\mathcal{W}) \times \mathcal{O}_{0}(\overline{\mathbb{C}} \backslash \mathcal{W})$. Alors $\left(f_{n}\right)$ est une base de $\mathcal{O}(\mathcal{W})$ si et seulement si : 
(i) $\left(f_{n}\right)$ est totale dans $\mathcal{O}(\mathcal{W})$.

(ii) $\left\{f_{n}(z) \varphi_{n}(\xi)\right\}$ est une partie bornée de $\mathcal{O}(\mathcal{W} \times(\overline{\mathbb{C}} \backslash \mathcal{W}))$.

C'est une conséquence immédiate de l'important critère de Dynin et Mityagin ([M], Th. 9).

(3) Maintenant on va utiliser la proposition avec $D, E$ comme dans l'énoncé du théorème 1 et $G=\overline{\mathbb{C}} \backslash E, F=\overline{\mathbb{C}} \backslash D$. Il résulte de la proposition et du lemme précédent que $\left\{f_{n}(z) \varphi_{n}(\xi)\right\}$ est une partie bornée de $\mathcal{O}(\Omega)$, où $\Omega=\{(z, \xi) \in D \times G: \omega(E, D, z)+\omega(F, G, \xi)<1\}$.

Pour plus de clarté, $\omega$ (resp. $\widetilde{\omega}$ ) représentera dorénavant la fonction extrémale associée au couple $(E, D)$ (resp. $(F, G)$ ).

Soit $\alpha \in] 0,1\left[\right.$ tel que $\partial D_{\alpha} \subset D \backslash E$; montrons que $D_{\alpha} \times\left(\overline{\mathbb{C}} \backslash D_{\alpha}\right) \subset \Omega$.

$(\bullet)$ Sur $D \backslash E, \omega+\widetilde{\omega} \equiv 1$.

En effet, il est facile de voir que la fonction $\omega+\widetilde{\omega}$, harmonique sur $D \backslash E$, admet 1 comme valeur frontière quasi-partout sur $\partial(D \backslash E)$. C'est donc la constante 1 .

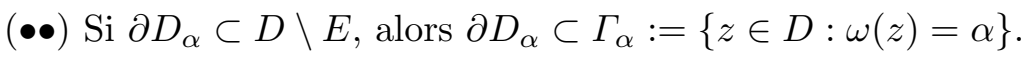

Soit $a \in \partial D_{\alpha}$; il est évident que $\omega(a) \geq \alpha$. Supposons $\omega(a)>\alpha$. Par continuité de $\omega$ sur $D \backslash E$ il existe alors un voisinage de $a$ sur lequel $\omega(z)>\alpha$, c'est donc un voisinage de $a \in \bar{D}_{\alpha}$ qui ne rencontre pas $D_{\alpha}$, ce qui est absurde et par conséquent $\omega(a)=\alpha$.

Fixons maintenant $\alpha$ tel que $\partial D_{\alpha} \subset D \backslash E$ et soit $(z, \xi) \in D_{\alpha} \times\left(\overline{\mathbb{C}} \backslash D_{\alpha}\right)$. On a par le principe du maximum

$$
\widetilde{\omega}(\xi) \leq \sup _{\xi \in \partial D_{\alpha}} \widetilde{\omega}(\xi) .
$$

En raison de $(\bullet)$ et $(\bullet \bullet)$ le second membre est égal à $1-\alpha$. Donc $\omega(z)+\widetilde{\omega}(\xi)$ $<\alpha+1-\alpha=1$, d'où la relation $D_{\alpha} \times\left(\overline{\mathbb{C}} \backslash D_{\alpha}\right) \subset \Omega$.

$\left\{f_{n} \varphi_{n}\right\}$ étant une partie bornée de $\mathcal{O}(\Omega)$, est donc une partie bornée de $\mathcal{O}\left(D_{\alpha} \times\left(\mathbb{C} \backslash D_{\alpha}\right)\right)$. En outre, il est facile de voir que $\widehat{\mathcal{K}}_{\mathcal{S H}(D)} \Subset D_{\alpha}$ pour tout compact $\mathcal{K}$ de $D_{\alpha}\left(\widehat{\mathcal{K}}_{\mathcal{S H}(D)}\right.$ désignant l'enveloppe de $\mathcal{K}$ par rapport aux fonctions sousharmoniques sur $D) ; D_{\alpha}$ est donc $\mathcal{O}(D)$-convexe et forme une paire de Runge avec $D$, donc $\left(f_{n}\right)$ est totale dans $\mathcal{O}\left(D_{\alpha}\right)$, ce qui achève la démonstration.

Corollaire (du théorème 1). Si l'on suppose en plus que D soit à bord régulier pour le problème de Dirichlet, alors toute base commune $\left(f_{n}\right)$ de $\mathcal{O}(D)$ et $\mathcal{O}(E)$ est une base de $\mathcal{O}\left(D_{\alpha}\right)$ et $\mathcal{O}\left(\bar{D}_{\alpha}\right)$, pour tout $\left.\alpha \in\right] \alpha_{0}, 1[$, avec $\alpha_{0}=\sup _{E} \omega$.

Démonstration. Remarquons que $\alpha_{0}<1$ et $D_{\alpha} \Subset D, \forall \alpha<1(E$ compact non polaire dans $D$ régulier). Soit $\alpha \in] \alpha_{0}, 1[$. 
(i) $\partial D_{\alpha} \subset D \backslash E$.

Supposons $\partial D_{\alpha} \not \subset D \backslash E$. Alors $\partial D_{\alpha} \cap \partial(D \backslash E) \neq \emptyset$; on a donc $\partial D_{\alpha} \cap E \neq$ $\emptyset$, car $D_{\alpha} \Subset D$. Soit $a \in \partial D_{\alpha} \cap E$. On a $\omega(a) \leq \alpha_{0}<\alpha$ : impossible, car $\partial D_{\alpha} \cap D_{\alpha}=\emptyset$. D'où (i).

(ii) $\partial D_{\alpha}=\Gamma_{\alpha}:=\{z \in D: \omega(z)=\alpha\}$.

D'après la preuve du théorème 1 , (i) implique $\partial D_{\alpha} \subset \Gamma_{\alpha}$. Pour l'inclusion inverse, il suffit de prouver que tout point $a \in \Gamma_{\alpha}$ est adhérent à $D_{\alpha}$. Soit $\Delta$ une boule arbitraire de centre $a$. Prouvons que $\Delta \cap D_{\alpha} \neq \emptyset$. On peut supposer $\Delta \subset D \backslash E$; si $\Delta \cap D_{\alpha}=\emptyset$, $\omega$ serait $\geq \alpha$ sur $\Delta$ et $\omega(a)=\alpha$, elle serait donc constante sur la composante connexe $\mathcal{C}$ de $D \backslash E$ contenant $a$. Puisque $(E, D)$ est une paire de Runge, $\partial \mathcal{C} \cap \partial D$ est non vide. En un point $b \in \partial \mathcal{C} \cap \partial D$ on a

$$
\lim _{\substack{\xi \rightarrow b \\ \xi \in D}} \omega(\xi)=1 \quad \text { et } \quad \lim _{\substack{\xi \rightarrow b \\ \xi \in \mathcal{C}}} \omega(\xi)=\alpha<1,
$$

ce qui est absurde, d'où (ii).

(iii) $\left(f_{n}\right)$ est bien une base de $\mathcal{O}\left(D_{\alpha}\right)$, d'après le théorème 1 et (i). Il est plus facile de voir, en raison de (ii), que la famille $\left\{D_{\beta}\right\}_{\beta>\alpha}$ forme une base de voisinages ouverts de $\bar{D}_{\alpha}$ dans $D ;\left(f_{n}\right)$ étant une base de $\mathcal{O}\left(D_{\beta}\right)$ pour tout $\beta \in] \alpha, 1$, est encore une base de $\mathcal{O}\left(\bar{D}_{\alpha}\right)=\operatorname{limind}_{\beta \rightarrow \alpha, \beta>\alpha} \mathcal{O}\left(D_{\beta}\right)$.

2. Démonstration du théorème 2. En raison de la convention faite dans l'introduction, $D$ est un domaine simplement connexe $\neq \mathbb{C}$ et $E$ est un compact non polaire dans $D$. Supposons que $\omega \not \equiv 0$ sur $E$; alors $\lambda:=$ $\left.\alpha_{0}=\sup _{E} \omega \in\right] 0,1[$. Soit $a$ un point frontière de $E$ tel que $\omega(a)=\lambda$. $E$ est régulier en au moins un point $b$ de sa frontière; puisque $\lambda>\omega(b)=0$, on peut trouver un disque ouvert $\mathcal{U}$ centré en $b$ suffisamment petit pour que

$$
\omega(z)<\lambda, \quad \forall z \in \mathcal{U} .
$$

Soit $f \in \mathcal{O}(E)$; il existe donc un voisinage ouvert $\mathcal{V}$ de $E$ sur lequel $f$ est holomorphe, et puisque $\left(f_{n}\right)$ est une base de $\mathcal{O}(E)$ et donc une base absolue (cf. Mityagin $[\mathrm{M}]$ ), on peut trouver une suite de scalaires $\left(b_{n}\right)$ telle que la série $\sum b_{n} f_{n}$ converge normalement sur un voisinage ouvert $\mathcal{W}$ de $E(\mathcal{W} \subset \mathcal{V})$ et a pour somme $f$ sur $\mathcal{W}$. On peut donc choisir $\mu \in] \lambda, 1[$ suffisamment proche de $\lambda$ pour que $\left(D \backslash \bar{D}_{\mu}\right) \cap \mathcal{W}$ soit non vide. D'après le corollaire du théorème $1,\left(f_{n}\right)$ est une base commune aux espaces $\mathcal{O}\left(\bar{D}_{\mu}\right)$ et $\mathcal{O}(D)$. Comme nous l'avons remarqué dans la démonstration du corollaire, $\Gamma_{\mu}=\partial D_{\mu} ;$ la frontière de $\bar{D}_{\mu}$ est donc analytique réelle, et le couple $\left(\bar{D}_{\mu}, D\right)$ est régulier. De plus, $\bar{D}_{\mu}$ est polynomialement convexe car la paire $\left(\bar{D}_{\mu}, D\right)$ est de Runge et $D$ est un domaine simplement connexe : $D \backslash \bar{D}_{\mu}$ est donc 
connexe. Nous sommes donc en mesure d'appliquer les résultats de Dragilev et Nguyen Thanh Van ([N], pp. 227-229).

$(\mathcal{A})$ Si l'on pose $\varrho=\exp (1 / \Phi)$ où $\Phi$ est le flux de la fonction $h=$ $\omega\left(\bar{D}_{\mu}, D, \cdot\right)$ à travers tout contour séparant $\bar{D}_{\mu}$ et $\overline{\mathbb{C}} \backslash D$, alors il existe une suite $\left(\lambda_{n}\right)$ de nombres $>0$ et une bijection $\pi$ de $\mathbb{N}$ sur $\mathbb{N}$ telle que la suite $g_{n}=\lambda_{n} f_{\pi(n)}$ possède les propriétés suivantes :

(i) $\lim _{n \rightarrow \infty}\left\|g_{n}\right\|_{\widetilde{D}_{\alpha}}^{1 / n}=\varrho^{\alpha}$, avec $\widetilde{D}_{\alpha}:=\{z \in D: h(z)<\alpha\}$,

(ii) $\lim _{n \rightarrow \infty}\left\|g_{n}\right\|_{\Delta}^{1 / n}=\varrho^{\alpha(\Delta)}$, pour tout disque $\Delta \Subset D \backslash \bar{D}_{\mu}$, avec

$$
\alpha(\Delta):=\sup _{\Delta} h
$$

Revenons maintenant à notre série $\sum b_{n} f_{n}$. Puisqu'elle converge normalement sur un voisinage ouvert $\mathcal{W}$ de $E$ et a pour somme $f$, il en est de même pour la série $\sum a_{n} g_{n}$ avec $a_{n}=b_{\pi(n) / \lambda_{n}}$. Choisissons un disque $\Delta \Subset\left(D \backslash D_{\mu}\right) \cap \mathcal{W}$. On a

$$
\left|a_{n}\right| \cdot\left\|g_{n}\right\|_{\Delta} \leq M, \quad \forall n \in \mathbb{N}\left(M=C^{t e}\right),
$$

donc d'après (ii),

$$
\limsup _{n \rightarrow \infty}\left|a_{n}\right|^{1 / n} \leq \limsup _{n \rightarrow \infty} \frac{1}{\left\|g_{n}\right\|_{\Delta}^{1 / n}}=\varrho^{-\alpha(\Delta)} .
$$

Choisissons alors $\beta$ dans ]0, $\alpha(\Delta)[$. L'inégalité précédente et (i) entraînent la normale convergence de la série $\sum a_{n} g_{n}$ sur $\widetilde{D}_{\beta}$ qui contient $\mathcal{U}$ (rappelons que $\sup _{\mathcal{U}} \omega<\lambda$; ceci entraîne $\mathcal{U} \subset D_{\mu}$ et d'autre part $\widetilde{D}_{\beta}$ est un voisinage de $\bar{D}_{\mu}$ ). Ainsi $\sum a_{n} g_{n}$ est normalement convergente sur $\mathcal{U}, f$ est donc analytiquement prolongeable à $\mathcal{U}$.

On a donc démontré que toute fonction de $\mathcal{O}(E)$ est analytiquement prolongeable à $\mathcal{U}$, ce qui est impossible.

Remarques. (i) Notre démonstration s'appuie sur le corollaire du théorème 1 et la démarche du chapitre 4 de $[\mathrm{N}]$, inspirée elle-même par un travail de Dragilev [D]. L'énoncé $(\mathcal{A})$ est le cas particulier du

ThÉORÈme. Soient $\Omega$ un domaine de $\mathbb{C}, \mathcal{X}$ un compact dans $\Omega$ tel que le couple $(\mathcal{X}, \Omega)$ soit régulier et que $\Omega \backslash \mathcal{X}$ soit connexe. Soit $\Phi$ le flux de $\omega(\mathcal{X}, \Omega, \cdot)$ à travers tout contour séparant $\mathcal{X}$ et $\overline{\mathbb{C}} \backslash \Omega$. Alors si $\mathcal{O}(\mathcal{X})$ et $\mathcal{O}(\Omega)$ possèdent une base commune $\left(f_{n}\right)$, il existe une suite $\left(\lambda_{n}\right)$ de nombres $>0$ et une bijection $\pi$ de $\mathbb{N}$ sur lui-même telle que la suite de terme général $g_{n}=\lambda_{n} f_{\pi(n)}$ vérifie, pour tout disque $\Delta \Subset \Omega \backslash \mathcal{X}$,

$$
\lim _{n \rightarrow \infty}\left\|g_{n}\right\|_{\Delta}^{1 / n}=R^{\alpha(\Delta)}
$$

avec $R=\exp (1 / \Phi)$ et $\alpha(\Delta):=\sup _{\Delta} \omega(\mathcal{X}, \Omega, \cdot$ ) (pour la démonstration cf. [N], pp. 227-229). 
En conséquence on voit que le théorème 2 reste valable sous une hypothèse moins restrictive : Au lieu de supposer $D$ domaine simplement connexe $\neq \mathbb{C}$, on peut supposer :

- $D$ domaine régulier,

- il existe $\left.\left.\mu_{0} \in\right] \lambda, 1\right]$ tel que $D_{\mu_{0}} \backslash \bar{D}_{\mu}$ soit connexe pour des valeurs de $\mu>\lambda$ aussi proches de $\lambda$ que l'on veut $\left(\lambda:=\sup _{E} \omega(E, D, \cdot)\right)$.

(ii) Le théorème 2 n'est apparemment pas nouveau. On trouve dans l'article de Zakharyuta et Kadampatta [ZK] l'énoncé suivant plus général :

[ZK] Soient $D$ un ouvert régulier de $\mathbb{C}$ et $E$ un compact de D. Si $\mathcal{O}(E)$ et $\mathcal{O}(D)$ possèdent une base commune et, si pour une composante connexe $\Delta$ de $D, \widetilde{E}=\Delta \cap E$ est non polaire, alors $\omega(E, D, \cdot) \equiv 0$ sur $\widetilde{E}$.

En raison de coupures draconniennes (pour raccourcir), ce travail est très difficile à lire. Il est basé sur l'assertion suivante : Si $\left(f_{n}\right)$ est une base commune de $\mathcal{O}(E)$ et $\mathcal{O}(D)$, alors il existe des espaces Hilbertiens $\mathcal{H}_{0}$ et $\mathcal{H}_{1}$ tels que :

(a) $\mathcal{O C}(\overline{\mathbb{C}} \backslash D) \rightarrow \mathcal{H}_{1} \rightarrow \mathcal{O}(D) \rightarrow \mathcal{O}(E) \rightarrow \mathcal{H}_{0} \rightarrow \mathcal{O C}(E)$ (la flèche $\rightarrow$ signifie injection linéaire continue, la dernière n'est pas nécessairement injective),

(b) $\left(f_{n}\right)$ est une base orthogonale de $\mathcal{H}_{0}$ et $\mathcal{H}_{1}$.

D'après V. P. Zakharyuta (communication orale), cette assertion peut être prouvée par des considérations de nucléarité. (a) résulte de ([M], Prop. $3 a)$, cependant (b) nous paraît être un résultat fort qui mériterait une preuve.

$\mathrm{Au}$ cours de la démonstration de l'énoncé $[\mathrm{ZK}]$ ci-dessus, les auteurs semblent supposer que pour un certain $\beta>0$, valeur prise par $\omega$ en un point irrégulier de $E, \Delta \backslash \bar{\Delta}_{\beta}$ est connexe, où

$$
\Delta_{\beta}=\text { Intérieur de }[\widetilde{E} \cup\{z \in \Delta: \omega(E, D, \cdot)<\beta\}] .
$$

\section{Bibliographie}

Outre les références citées dans le texte, on signale le travail de pionnier de V. D. Erokhin ([E1], développé dans [E2]) et celui de T. Bagby où figure implicitement la première démonstration de l'existence d'une base commune pour $\mathcal{O}(D)$ et $\mathcal{O}(E)$ lorsque $(E, D)$ est une paire de Runge régulière.

[B] T. Bagby, Interpolation by rational functions, Duke Math. J. 36 (1969), 95-104.

[D] M. M. Dragilev, Extendable bases of analytic functions, Amer. Math. Soc. Transl. (2) 43 (1964), 267-280. 
[E1] V. D. Erokhin, Sur les transformations conformes de couronnes et la base fondamentale de l'espace des fonctions analytiques sur un voisinage élémentaire d'un continuum arbitraire, Dokl. Akad. Nauk SSSR 120 (1958), 689-692.

[E2] - Best linear approximation of functions analytically continuable from a continuum to a given region, Russian Math. Surveys 23 (1968), 96-122.

[M] B. S. Mityagin, Approximative dimension and bases in nuclear Fréchet spaces, Russian Math. Surveys 16 (1961), 59-127.

[N] Nguyen Thanh Van, Bases de Schauder dans certains espaces de fonctions holomorphes, Ann. Inst. Fourier (Grenoble) 22 (2) (1972), 169-253.

[NS] Nguyen Thanh Van and J. Siciak, Fonctions plurisousharmoniques extrémales et systèmes doublement orthogonaux de fonctions analytiques, Bull. Sci. Math. 115 (1991), 235-244.

[NZ] Nguyen Thanh Van and A. Zeriahi, Une extension du Théorème de Hartogs sur les fonctions séparément analytiques, dans : Analyse Complexe Multivariable, récents développements, A. Meril (éd.), Editel s.n.c, Rende, 1991, 185-194.

[S] J. Siciak, Separately analytic functions and envelopes of holomorphy of some lower-dimensional subsets of $\mathbb{C}^{n}$, Ann. Polon. Math. 22 (1969), 145-171.

[W] J. M. Whittaker, Leçons sur les séries de base de polynomes quelconques, Collection Borel 39, Gauthier-Villars, Paris, 1949.

[Z] V. P. Zakharyuta, Continuable bases in spaces of analytic functions of one and several complex variables, Siberian Math. J. 8 (1967), 204-216.

[ZK] V. P. Zakharyuta and S. N. Kadampatta, Existence of continuable bases in spaces of functions analytic in compacta, Math. Notes 27 (1980), 334-340.

LABORATOIRE D'ANALYSE

U.F.R. MIG

UNIVERSITÉ PAUL SABATIER

118, ROUTE DE NARBONNE

31062 TOULOUSE CEDEX, FRANCE

E-mail: LASSERE@CIX.CICT.FR 